Article

\title{
Analysis of the dynamics of avian influenza A(H7N9) epidemic model with re-infection
}

\author{
Abayomi Samuel OKE ${ }^{1, *}$ and Oluwafemi Isaac BADA ${ }^{2}$ \\ 1 Department of Mathematical Sciences, Adekunle Ajasin University, P.M.B. 001, Akungba Akoko, Ondo State, \\ Nigeria. \\ 2 Department of Mathematical Sciences, University of Benin, P.M.B. 1154, Benin City, Nigeria; \\ badaoluwafemiisaac@gmail.com \\ * Correspondence: okeabayomisamuel@gmail.com, abayomi.oke@aaua.edu.ng
}

Received: 13 May 2019; Accepted: 15 November 2019; Published: 7 December 2019.

\begin{abstract}
Since the emergence of the avian influenza A(H7N9) in the year 2013 in China, several researches have been carried out to investigate the spread. In this paper, a mathematical model describing the transmission dynamics of avian influenza $\mathrm{A}(\mathrm{H} 7 \mathrm{~N} 9)$ between human and poultry proposed by Li et al. [1] is modified by introducing re-infections into the susceptible human compartment. The method of next generation matrix is used to calculate the reproduction number. We also establish the local and global stability of the equilibria using Lyapunov functions. Finally, we use numerical simulations to validate our results.
\end{abstract}

Keywords: Avian influenza A(H7N9), reproduction number, Lyapunov functions, next generation matrix, re-infections.

MSC: 34D20, 34D20, 65P40, 37N25.

\section{Introduction}

A vian influenza refers to a group of viruses that are spread between birds and are grouped into Low A Pathogenicity Avian Influenza (LPAI) and Highly Pathogenicity Avian Influenza (HPAI). One novel LPAI is the avian influenza A(H7N9) which emerged in humans in Eastern China in February 2013 [2-8]. The transmission of avian influenza $\mathrm{A}(\mathrm{H} 7 \mathrm{~N} 9)$ occurs between poultry and poultry or between poultry and human but no case has been recorded yet about human to human transmission. Iwami et al. in [9] considered a dynamic model of avian influenza that might be transmitted by infected poultry and infected humans with variant avian influenza. Che et al. [10] studied a model of highly pathogenic avian influenza with saturated contact rate. Liu et al. [11] established a dynamical model of avian influenza A(H7N9) that can spread between poultry and poultry, poultry and human, and human and human to evaluate the impact of these measures on avian influenza A(H7N9) epidemic. From the available epidemiological and virological evidence, thus the likelihood of human-to-human transmission of the avian influenza $\mathrm{A}(\mathrm{H} 7 \mathrm{~N} 9)$ is low. Li et al. [1] investigated the mathematical model describing the transmission dynamics of avian influenza A(H7N9) between human and poultry, in which there is no human-to-human transmission. They however, ignored the possibility of human re-infection. In [12], Khan et al. proposed two models considering saturated incidence rate and psychological effect in the model. Zhang et al. [13] constructed an avian influenza A (H7N9) epidemic model with vaccination and seasonality to study the spread of avian influenza A (H7N9). Other works on influenza virus include the works of [14-16]. In this paper, we extend the model proposed by [1]. We consider the possibility of human re-infection which transfers every recovered human back into the susceptible class.

\section{The model}

According to [1], human have the risk of getting infected with avian influenza A(H7N9) largely because of their exposure to poultry. In [1], Li et al. further developed a mathematical model to investigate the spread of avian influenza A(H7N9) to human. The assumptions in the model include:

1. transmission of avian influenza A(H7N9) occurs only in two ways; firstly, between poultry and poultry and secondly, between poultry and human. It cannot transmit from human to human. 
2. the human population is classified each into Susceptible, Infected, and Recovered.

3. poultry in the farm and poultry in the market are classified into Susceptible and Infected.

Consider $S, I$, and $R$ denote Susceptible, Infected, and Recovered class respectively and the subscripts $h$, $f a$, and $m a$ denote human, farm and market respectively. The established model is given as:

$$
\left.\begin{array}{c}
\frac{d S_{f a}}{d t}=A_{a}-d_{a} S_{f a}-a S_{f a}-\beta_{a} S_{f a} I_{f a} \\
\frac{d I_{f a}}{d t}=\beta_{a} S_{f a} I_{f a}-d_{a} I_{f a}-a I_{f a}-\alpha_{a} I_{f a} \\
\frac{d S_{m a}}{d t}=a S_{f a}-\beta_{m} S_{m a} I_{m a}-d_{m} S_{m a} \\
\frac{d I_{m a}}{d t}=a I_{f a}+\beta_{m} S_{m a} I_{m a}-d_{m} I_{m a}-\alpha_{m} I_{m a} \\
\frac{d S_{h}}{d t}=A_{h}-\beta_{h} S_{h} I_{m a}-d_{h} S_{h} \\
\frac{d I_{h}}{d t}=\beta_{h} S_{h} I_{m a}-d_{h} I_{h}-r I_{h}-\alpha_{h} I_{h} \\
\frac{d R_{h}}{d t}=r I_{h}-d_{h} R_{h} .
\end{array}\right\}
$$

where $A_{h}$ and $A_{a}$ represent the birth rates of human and poultry respectively, and $d_{h}, d_{a}$ and $d_{m}$ indicate the natural mortality rates of human, poultry of farms, and poultry of markets respectively.

Here $\alpha_{h}, \alpha_{a}$ and $\alpha_{m}$ are the disease-related death rates of infected human, infected poultry of farms, and infected poultry of markets respectively, $\beta_{a}$ is the transmission coefficient from infective poultry of farms to susceptible poultry of farms, $\beta_{m}$ is the contact rate from infective poultry of markets to susceptible poultry of markets, $\beta_{h}$ is the transmission rate from infected poultry of markets to susceptible human, $r$ is the recovery rate of infected human and $a$ is the proportion of poultry from farms to markets. All the parameters are non-negative.

It is worth noting that this model ignores the possibility of re-infection. We modify this model by assuming that a recovered individual who comes in contact with infective poultry can become infected again. This implies that any recovered individual moves out of the recovered compartment and thus, the equation for the recovered compartment becomes:

$$
\frac{d R_{h}}{d t}=r I_{h}-R_{h}
$$

It is also important to note that some of the recovered individual may die naturally. This means that the population of the recovered individuals entering back into the Susceptible class is $\left(1-d_{h}\right) R_{h}$ and the equation for the Susceptible class becomes

$$
\frac{d S_{h}}{d t}=A_{h}-\beta_{h} S_{h} I_{m a}-d_{h} S_{h}+\left(1-d_{h}\right) R_{h} .
$$

Combining these equations with the existing ones gives the proposed model as

$$
\begin{aligned}
& \frac{d S_{f a}}{d t}=A_{a}-d_{a} S_{f a}-a S_{f a}-\beta_{a} S_{f a} I_{f a} \\
& \frac{d I_{f a}}{d t}=\beta_{a} S_{f a} I_{f a}-d_{a} I_{f a}-a I_{f a}-\alpha_{a} I_{f a} \\
& \frac{d S_{m a}}{d t}=a S_{f a}-\beta_{m} S_{m a} I_{m a}-d_{m} S_{m a} \\
& \frac{d I_{m a}}{d t}=a I_{f a}+\beta_{m} S_{m a} I_{m a}-d_{m} I_{m a}-\alpha_{m} I_{m a} \\
& \frac{d S_{h}}{d t}=A_{h}-\beta_{h} S_{h} I_{m a}-d_{h} S_{h}+\left(1-d_{h}\right) R_{h} \\
& \frac{d I_{h}}{d t}=\beta_{h} S_{h} I_{m a}-d_{h} I_{h}-r I_{h}-\alpha_{h} I_{h} \\
& \frac{d R_{h}}{d t}=r I_{h}-R_{h} .
\end{aligned}
$$




\subsection{Non-negativity Conditions}

In order to establish the validity of the model, we establish the non-negativity conditions for the farm poultry population, market poultry population and the human population. Let

$$
\begin{aligned}
N_{f a}(t) & =S_{f a}(t)+I_{f a}(t) \\
N_{m a}(t) & =S_{m a}(t)+I_{m a}(t) \\
N_{h}(t) & =S_{h}(t)+I_{h}(t)+R_{h}(t)
\end{aligned}
$$

where $N_{f a}, N_{m a}$ and $N_{h}$ represent the total population of poultry in farm, total population of poultry in market and total human population respectively. By putting (9-11) into (2-8), Equations (2-8) reduce to the following system of three linear ordinary differential equations

$$
\begin{aligned}
& \frac{d N_{f a}}{d t}=A_{a}-\left(d_{a}+a\right) N_{f a}-\alpha_{a} I_{f a} \leq A_{a}-\left(d_{a}+a\right) N_{f a} \\
& \frac{d N_{m a}}{d t}=a N_{f a}-d_{m} N_{m a}-\alpha_{m} I_{m a} \leq a N_{f a}-d_{m} N_{m a} \\
& \frac{d N_{h}}{d t}=A_{h}-d_{h} N_{h}-\alpha_{h} I_{h} \leq A_{h}-d_{h} N_{h}
\end{aligned}
$$

Then, from (12), it follows that

$$
N_{f a}(t) \leq \frac{A_{a}}{a+d_{a}}+\left(N_{f a}(0)-\frac{A_{a}}{a+d_{a}}\right) e^{-\left(a+d_{a}\right) t}
$$

and so

$$
\lim _{t \rightarrow \infty} N_{f a}(t) \leq \frac{A_{a}}{a+d_{a}} .
$$

In the same way, it can be obtained from (13) and (14) that

$$
\lim _{t \rightarrow \infty} N_{m a}(t) \leq \frac{a A_{a}}{d_{m}\left(a+d_{a}\right)}, \text { and } \lim _{t \rightarrow \infty} N_{h}(t) \leq \frac{A_{h}}{d_{h}} .
$$

The feasible region of the system (2-8) is

$$
\Omega=\left\{\left(S_{f a}, I_{f a}, S_{m a}, I_{m a}, S_{h}, I_{h}, R_{h}\right) \in \mathbb{R}_{+}^{7}: N_{f a} \leq \frac{A_{a}}{a+d_{a}}, N_{m a} \leq \frac{a A_{a}}{d_{m}\left(a+d_{a}\right)}, N_{h} \leq \frac{A_{h}}{d_{h}}\right\} .
$$

\section{The Existence of Equilibria}

The reproduction number is calculated in this section and we further show the existence of the positive equilibrium. It is easy to see from (2-8), that the disease-free equilibrium is $E^{0}=\left(S_{f a^{\prime}}^{0}, 0, S_{m a}^{0}, 0, S_{h^{\prime}}^{0} 0,0\right)$, where

$$
S_{f a}^{0}=\frac{A_{a}}{a+d_{a}}, S_{m a}^{0}=\frac{a A_{a}}{d_{m}\left(a+d_{a}\right)}, \text { and } S_{h}^{0}=\frac{A_{h}}{d_{h}} .
$$

Using the method proposed by [17], we can obtain the reproduction number by the next generation matrix

$$
R_{0}=\rho\left(F V^{-1}\right)
$$

where $R_{0}$ is the reproduction number, $\rho$ is the spectral radius of a matrix and

$$
F=\left[\frac{\partial f_{i}}{\partial x_{i}}\left(x_{0}\right)\right] \text { and } V=\left[\frac{\partial v_{j}}{\partial x_{j}}\left(x_{0}\right)\right] \text { and } x_{i} \text { are the relevant variables. }
$$

For the problem at hand, we have

$$
f=\left(\begin{array}{c}
\beta_{a} S_{f a} I_{f a} \\
\beta_{m} S_{m a} I_{m a} \\
\beta_{h} S_{h} I_{m a}
\end{array}\right), \quad v=\left(\begin{array}{c}
d_{a} I_{f a}+a I_{f a}+\alpha_{a} I_{f a} \\
d_{m} I_{m a}+\alpha_{m} I_{m a}-a I_{f a} \\
d_{h} I_{h}+r I_{h}+\alpha_{h} I_{h}
\end{array}\right)
$$


and consequently, we get reproduction number as

$$
R_{01}=\frac{A_{a} \beta_{a}}{\left(a+d_{a}\right)\left(a+d_{a}+\alpha_{a}\right)}, R_{02}=\frac{a A_{a} \beta_{m}}{d_{m}\left(a+d_{a}\right)\left(d_{m}+\alpha_{m}\right)} .
$$

Hence, the basic reproduction number of the system is

$$
R_{0}=\max \left\{R_{01}, R_{02}\right\} .
$$

Theorem 1. For system (2-8),

1. If $R_{01}>1, R_{02}>1$, there is the unique endemic equilibrium

$$
E^{* *}=\left(S_{f a}^{* *}, I_{f a}^{* *}, S_{m a}^{* *}, I_{m a}^{* *}, S_{h}^{* *}, I_{h}^{* *}, R_{h}^{* *}\right) .
$$

2. If $R_{01}<1, R_{02}>1$, then there is the unique boundary equilibrium

$$
E^{*}=\left(S_{f a}^{*}, 0, S_{m a}^{*}, S_{h}^{*}, I_{h}^{*}, R_{h}^{*}\right)
$$

Proof. There are only two possibilities for positive equilibrium. The first is when there are infective farm poultry (i.e. $I_{f a} \neq 0$ ) while the second is when there are no infective farm poultry $\left(I_{f a}=0\right)$.

1. Consider the first case where $I_{f a} \neq 0$, then it is obtained from equation (3) that

$$
S_{f a}^{* *}=\frac{a+\alpha_{a}+d_{a}}{\beta_{a}}
$$

Putting (17) in (2), we have

$$
I_{f a}^{* *}=\frac{\left(a+d_{a}\right)\left(R_{01}-1\right)}{\beta_{a}} .
$$

Since all parameters are positive and it is a necessary condition that $I_{f a}^{* *}>0$, then we require $R_{01}>1$ (from 18). Furthermore, suppose $I_{m a}=0$, then Equation (5) implies that $I_{f a}=0$ which contradicts the initial assumption. Hence, we are left to take $I_{m a} \neq 0$ and Equation (4) gives

$$
S_{m a}^{* *}=\frac{a S_{f a}^{* *}}{\beta_{m} I_{m a}^{* *}+d_{m}} .
$$

Substituting (19) into (5) gives

$$
a_{1} I_{m a}^{* * 2}+b_{1} I_{m a}^{* *}+c_{1}=0
$$

where

$$
\begin{aligned}
& a_{1}=-\beta_{m}\left(d_{m}+\alpha_{m}\right)<0 \\
& b_{1}=a \beta_{m} I_{f a}^{* *}+a \beta_{m} S_{f a}^{* *}-d_{m}^{2}-d_{m} \alpha_{m}, \\
& c_{1}=a d_{m} I_{f a}^{* *}>0
\end{aligned}
$$

which means that $\Delta=b_{1}^{2}-4 a_{1} c_{1}>0$ if $R_{02}>1$ and hence, there is a unique positive root

$$
I_{m a}^{* *}=\frac{-a I_{f a}^{* *}}{\beta_{m} S_{m a}^{* *}-d_{m}-\alpha_{m}}=\frac{-b_{1}-\sqrt{b_{1}^{2}-4 a_{1} c_{1}}}{2 a_{1}} .
$$

Substituting (22) into Equations (6-8) gives

$$
S_{h}^{* *}=\frac{A_{h}+\left(1-d_{h}\right) R_{h}^{* *}}{\beta_{h} I_{m a}^{* *}+d_{h}}, I_{h}^{* *}=\frac{\beta_{h} S_{h}^{* *} I_{m a}^{* *}}{d_{h}+r+\alpha_{h}}, R_{h}^{* *}=r I_{h}^{* *} .
$$


Therefore, we conclude that when $R_{01}>1$, and $R_{02}>1$, the endemic equilibrium

$$
E^{* *}=\left(S_{f a}^{* *}, I_{f a}^{* *}, S_{m a}^{* *}, I_{m a}^{* *}, S_{h}^{* *}, I_{h}^{* *}, R_{h}^{* *}\right)
$$

is obtained.

2. We now suppose $I_{f a}=0$ and this means Equation (3) becomes redundant. We obtain from Equation (2) that

$$
S_{f a}^{*}=\frac{A_{a}}{d_{a}+a}
$$

Substituting $I_{f a}=0$ into Equation (5) gives that either $I_{m a}=0$ or $S_{m a}=\frac{d_{m}+\alpha_{m}}{\beta_{m}}$ but since $I_{m a} \neq 0$ then we are left with

$$
S_{m a}^{*}=\frac{d_{m}+\alpha_{m}}{\beta_{m}}
$$

Putting (24) and (25) into (4), we have

$$
I_{m a}^{*}=\frac{a S_{f a}^{*}-d_{m} S_{m a}^{*}}{\beta_{m} S_{m a}^{*}}=\frac{a A_{a}}{\left(d_{m}+\alpha_{m}\right)\left(a+d_{a}\right)}-\frac{d_{m}}{\beta_{m}}=\frac{d_{m}\left(R_{02}-1\right)}{\beta_{m}},
$$

from which it is required that $R_{02}>1$. Substituting (26) into Equations (6-8), we finally have

$$
S_{h}^{*}=\frac{A_{h}+\left(1-d_{h}\right) R_{h}^{*}}{\beta_{h} I_{m a}^{*}+d_{h}}, I_{h}^{*}=\frac{\beta_{h} S_{h}^{*} I_{m a}^{*}}{d_{h}+r+\alpha_{h}}, R_{h}^{*}=r I_{h}^{*}
$$

where $R_{01}<1$. Hence, when $R_{01}<1$ and $R_{02}>1$, the boundary equilibrium

$$
E^{*}=\left(S_{f a}^{*}, 0, S_{m a}^{*}, S_{h}^{*}, I_{h}^{*}, R_{h}^{*}\right)
$$

is obtained.

\section{Stability of equilibria}

It has been established in the previous section that there exists disease-free and positive equilibria. We further investigate the stability of this equilibria.

\subsection{Stability of the disease-free equilibrium}

Theorem 2. Let $E^{0}=\left(S_{f a^{\prime}}^{0}, 0, S_{m a}^{0}, 0, S_{h^{\prime}}^{0}, 0,0\right)$ be the disease-free equilibrium of system (2-8).

1. If $R_{0}<1$, then $E^{0}$ is locally asymptotically stable;

2. If $R_{0}>1$, then $E^{0}$ is unstable.

Proof. The Jacobian matrix at the disease-free equilibrium $E^{0}$ is

$$
J_{E^{0}}=\left(\begin{array}{ccccccc}
-d_{a}-a & -\beta_{a} S_{f a}^{0} & 0 & 0 & 0 & 0 & 0 \\
0 & \beta_{a} S_{f a}^{0}-d_{a}-a-\alpha_{a} & 0 & 0 & 0 & 0 & 0 \\
a & 0 & -d_{m} & -\beta_{a} S_{m a}^{0} & 0 & 0 & 0 \\
0 & a & 0 & \beta_{m} S_{m a}^{0}-d_{m}-\alpha_{m} & 0 & 0 & 0 \\
0 & 0 & 0 & -\beta_{h} S_{h}^{0} & -d_{h} & 0 & 1-d_{h} \\
0 & 0 & 0 & \beta_{h} S_{h}^{0} & 0 & -d_{h}-\alpha_{h}-r & 0 \\
0 & 0 & 0 & 0 & 0 & r & -1
\end{array}\right)
$$

The characteristics equation of the Jacobian matrix is

$$
\begin{aligned}
& (\lambda+1)\left(\lambda+d_{h}\right)\left(\lambda+d_{m}\right)\left(\lambda+d_{a}+a\right)\left(\lambda+d_{h}+r+\alpha_{h}\right) \times \\
& \left(\lambda+d_{a}+a+\alpha_{a}-\beta_{a} S_{f a}^{0}\right)\left(\lambda+\alpha_{m}+d_{m}-\beta_{m} S_{m a}^{0}\right)=0
\end{aligned}
$$


and the eigenvalues are

$$
\begin{aligned}
& \lambda_{1}=-1, \lambda_{2}=-d_{h}, \lambda_{3}=-d_{m}, \lambda_{4}=-\left(d_{a}+a\right), \lambda_{5}=-\left(d_{h}+\alpha_{h}+r\right), \\
& \lambda_{6}=-\left(d_{a}+a+\alpha_{a}\right)+\frac{\beta_{a} A_{a}}{a+d_{a}}=\left(R_{01}-1\right)\left(d_{a}+a+\alpha_{a}\right)<0 \text { if } R_{01}<1, \\
& , \lambda_{7}=-\left(d_{m}+\alpha_{m}\right)+\frac{\beta_{m} a A_{a}}{d_{m}\left(a+d_{a}\right)}=\left(R_{02}-1\right)\left(d_{m}+\alpha_{m}\right)<0 \text { if } R_{02}<1 .
\end{aligned}
$$

Hence, all eigenvalues have negative real parts if $R_{01}<1$ and $R_{02}<1$ and consequently, $R_{0}=$ $\max \left\{R_{01}, R_{02}\right\}<1$ (see [18]). Thus, the disease-free equilibrium $E_{0}$ is locally asymptotically stable if $R_{0}<1$.

Theorem 3. For system (5), if $R_{01}<1$, the disease-free equilibrium $E^{0}$ is globally asymptotically stable.

Proof. The proof shall be constructed by taking the three different subsystems one after the other

1. Poultry subsystem in farms: Define a Lyapunov function for the poultry subsystem in farms

$$
V_{11}=S_{f a}-S_{f a}^{0}-S_{f a}^{0} \ln \left(\frac{S_{f a}}{S_{f a}^{0}}\right)+I_{f a},
$$

and then the derivative of $V_{11}$ is

$$
\frac{d V_{11}}{d t}=\frac{S_{f a}-S_{f a}^{0}}{S_{f a}} \frac{d S_{f a}}{d t}+\frac{d I_{f a}}{d t}
$$

and substituting (16), (2) and 3 into (29), we have

$$
\begin{aligned}
\frac{d V_{11}}{d t} & =-\frac{a+d_{a}}{S_{f a}}\left(S_{f a}-S_{f a}^{0}\right)^{2}+\beta_{a} S_{f a}^{0} I_{f a}-\left(d_{a}+a+\alpha_{a}\right) I_{f a} \\
& =-\frac{a+d_{a}}{S_{f a}}\left(S_{f a}-S_{f a}^{0}\right)^{2}+\left(d_{a}+a+\alpha_{a}\right)\left(R_{01}-1\right) I_{f a} .
\end{aligned}
$$

Clearly, $\frac{d V_{11}}{d t} \leq 0$ if $R_{01}<1$. We now have that

$$
\Omega_{1}=\left\{\left(S_{f a}, I_{f a}\right) \in \mathbb{R}_{+}^{2}: \frac{d V_{11}}{d t}=0\right\}=\left\{\left(S_{f a}, I_{f a}\right) \in \mathbb{R}_{+}^{2}: S_{f a}=S_{f a}^{0} I_{f a}=0\right\}=\left\{E_{f a}^{0}\right\},
$$

which according to Lassale's invariance principle, $E_{f a}^{0}$ is globally asymptotically stable $[19,20]$.

2. Poultry subsystem of markets: Poultry subsystem of markets with the avian components of farms already at the disease-free steady state is

$$
\begin{aligned}
& \frac{d S_{m a}}{d t}=a S_{f a}^{0}-\beta_{m} S_{m a} I_{m a}-d_{m} S_{m a} \\
& \frac{d I_{m a}}{d t}=\beta_{m} S_{m a} I_{m a}-d_{m} I_{m a}-\alpha_{m} I_{m a} .
\end{aligned}
$$

We define a Lyapunov function as

$$
V_{12}=S_{m a}-S_{m a}^{0}-S_{m a}^{0} \ln \left(\frac{S_{m a}}{S_{m a}^{0}}\right)+I_{m a}
$$

It follows that

$$
\frac{d V_{12}}{d t}=\frac{S_{m a}-S_{m a}^{0}}{S_{m a}} \frac{d S_{m a}}{d t}+\frac{d I_{m a}}{d t}
$$

Replacing $\frac{d S_{m a}}{d t}$ and $\frac{d I_{m a}}{d t}$ with equations (32) and (33) respectively, then substituting $S_{f a}^{0}=\frac{A_{a}}{a+d_{a}}$ and $d_{m} S_{m a}^{0}=a \frac{A_{a}}{d_{a}+a}$ from 16 and rearranging, we have

$$
\frac{d V_{12}}{d t}=-\frac{d_{m}}{S_{m a}}\left(S_{m a}-S_{m a}^{0}\right)^{2}+\left(d_{m}+\alpha_{m}\right)\left(R_{02}-1\right) I_{m a}
$$


from which $\frac{d V_{12}}{d t} \leq 0$ if $R_{02}<1$. Thus,

$$
\Omega_{2}=\left\{\left(S_{m a}, I_{m a}\right) \in \mathbb{R}_{+}^{2}: \frac{d V_{12}}{d t}=0\right\}=\left\{\left(S_{m a}, I_{m a}\right) \in \mathbb{R}_{+}^{2}: S_{m a}=S_{m a}^{0}, I_{m a}=0\right\}=\left\{E_{m a}^{0}\right\} .
$$

According to Lasalle's invariance principle $([19,20]), E_{m a}^{0}$ is globally asymptotically stable.

3. Human subsystem: Finally, considering the human subsystem with the avian components already at the disease-free steady state

$$
\left.\begin{array}{c}
\frac{d S_{h}}{d t}=A_{h}-d_{h} S_{h}+R_{h}\left(1-d_{h}\right), \\
\frac{d I_{h}}{d t}=-d_{h} I_{h}-r I_{h}-\alpha_{h} I_{h} \\
\frac{d R_{h}}{d t}=r I_{h}-R_{h}
\end{array}\right\}
$$

We define a Lyapunov function

$$
V_{13}=S_{h}-S_{h}^{0}-S_{h}^{0} \ln \frac{S_{h}}{S_{h}^{0}}+I_{h}+R_{h}
$$

and then the derivative of $V_{13}$ along the solutions of system (34) is

$$
\frac{d V_{13}}{d t}=\frac{S_{h}-S_{h}^{0}}{S_{h}} \frac{d S_{h}}{d t}+\frac{d I_{h}}{d t}+\frac{d R_{h}}{d t}
$$

Substituting $A_{h}=d_{h} S_{h}^{0}$ and Equations (34) in Equation (35), we have

$$
\frac{d V_{13}}{d t}=-\frac{d_{h}}{S_{h}}\left(S_{h}-S_{h}^{0}\right)^{2}+\frac{S_{h}-S_{h}^{0}}{S_{h}}\left(1-d_{h}\right) R_{h}-\left(d_{h}+\alpha_{h}\right) I_{h}-R_{h} \leq 0,
$$

and, thus, $\Omega_{3}=\left\{\left(S_{h}, I_{h}, R_{h}\right) \in \mathbb{R}_{+}^{3}: \frac{d V_{13}}{d t}=0\right\}=\left\{\left(S_{h}, I_{h}, R_{h}\right) \in \mathbb{R}_{+}^{3}: S_{h}=S_{h}^{0}, I_{h}=0, R_{h}=0\right\}=$ $\left\{E_{h}^{0}\right\}$. According to Lasalle's invariance principle, $E_{h}^{0}$ is globally asymptotically stable.

\subsection{Stability of the boundary equilibrium and the endemic equilibrium}

The Jacobian matrix of system (2-8) is given as,

$$
J=\left(\begin{array}{ccccccc}
-d_{a}-a-\beta_{a} I_{f a} & -\beta_{a} S_{f a} & 0 & 0 & 0 & 0 & 0 \\
\beta_{a} I_{f a} & \beta_{a} S_{f a}-d_{a}-a-\alpha_{a} & 0 & 0 & 0 & 0 & 0 \\
a & 0 & -\beta_{m} I_{m a}-d_{m} & -\beta_{m} S_{m a} & 0 & 0 & 0 \\
0 & a & \beta_{m} I_{m a} & \beta_{m} S_{m a}-d_{m}-\alpha_{m} & 0 & 0 & 0 \\
0 & 0 & 0 & -\beta_{h} S_{h} & -\beta_{h} I_{m a}-d_{h} & 0 & 1-d_{h} \\
0 & 0 & 0 & \beta_{h} S_{h} & \beta_{h} I_{m a} & -d_{h}-r-\alpha_{h} & 0 \\
0 & 0 & 0 & 0 & 0 & r & -1
\end{array}\right) .
$$

The characteristics equation of the Jacobian matrix is:

$$
\begin{aligned}
& {\left[\left(\lambda+d_{a}+a+\beta_{a} I_{f a}\right)\left(\lambda+d_{a}+a+\alpha_{a}-\beta_{a} S_{f a}\right)+\beta_{a}^{2} S_{f a} I_{f a}\right]} \\
& \times\left[\left(\lambda+d_{m}+\beta_{m} I_{m a}\right)\left(\lambda+\alpha_{m}+d_{m}-\beta_{m} S_{m a}\right)+\beta_{m}^{2} S_{m a} I_{m a}\right] \\
& \times\left[\left(\lambda+d_{h}+\beta_{h} I_{m a}\right)\left(\lambda+d_{h}+r+\alpha_{h}\right)(\lambda+1)\right]=0
\end{aligned}
$$

Theorem 4. For system (5), if $R_{01}<1, R_{02}>1$, the boundary equilibrium $E^{*}$ is locally asymptotically stable; if $R_{01}>$ $1, R_{02}>1$, the endemic equilibrium $E^{* *}$ is locally asymptotically stable.

Proof. The proof is as follows; 
1. For the boundary equilibrium $E^{*}=\left(S_{f a}^{*}, 0, S_{m a}^{*}, I_{m a}^{*}, S_{h}^{*}, I_{h}^{*}, R_{h}^{*}\right)$, five of the eigenvalues are

$$
\begin{aligned}
& \lambda_{1}=-1, \lambda_{2}=-d_{h}-r-\alpha_{h}, \lambda_{3}=-d_{h}-\beta_{h} I_{m a}^{*} \\
& \lambda_{4}=-a-d_{a}, \lambda_{5}=-\alpha_{a}-a-d_{a}+\frac{\beta_{a} A_{a}}{a+d_{a}}=\left(\alpha_{a}+a+d_{a}\right)\left(R_{01}-1\right)
\end{aligned}
$$

and the remaining two eigenvalues $\lambda_{6}, \lambda_{7}$ depend on

$$
\lambda^{2}+\lambda d_{m}\left(R_{02}-1\right)+d_{m}\left(\alpha_{m}+d_{m}\right)\left(R_{02}-1\right)=0 .
$$

Hence, if $R_{01}<1$ and $R_{02}>1$, all eigenvalues have negative real parts.

2. For the endemic equilibrium $E^{* *}=\left(S_{f a}^{* *}, I_{f a}^{* *}, S_{m a}^{* *}, I_{m a}^{* *}, S_{h}^{* *}, I_{h}^{* *}, R_{h}^{* *}\right)$, three of the eigenvalues are

$$
\lambda_{1}=-1, \lambda_{2}=-d_{h}-r-\alpha_{h}, \lambda_{3}=-d_{h}-\beta_{h} I_{m a}^{* *}
$$

while $\lambda_{4}, \lambda_{5}$ are obtained from

$$
\lambda^{2}+\lambda R_{01}\left(d_{a}+a\right)+\left(d_{a}+a\right)\left(\alpha+a+d_{a}\right)\left(R_{01}-1\right)=0
$$

and the remaining two eigenvalues $\lambda_{6}, \lambda_{7}$ are obtained from

$$
\lambda^{2}+\lambda\left(2 d_{m}+\alpha_{m}-\beta_{m} S_{m a}^{* *}+\beta_{m} I_{m a}^{* *}\right)+\beta_{m} d_{m} I_{m a}^{* *}+\beta_{m} \alpha_{m} I_{m a}^{* *}+d_{m}\left(d_{m}+\alpha_{m}-\beta_{m} S_{m a}^{* *}\right)=0
$$

It can be obtained that $d_{m}+\alpha_{m}-\beta_{m} S_{m a}^{* *}>0$ by $I_{m a}^{* *}>0$. Hence, if $R_{01}>1, R_{02}>1$, all eigenvalues have negative real parts.

Theorem 5. For system (5),

1. if $R_{01}<1, R_{02}>1$, the boundary equilibrium $E^{*}$ is globally asymptotically stable,

2. if $R_{01}>1, R_{02}>1$, the endemic equilibrium $E^{* *}$ is globally asymptotically stable.

Proof. We consider the global stability of the boundary equilibrium and the endemic equilibrium.

1. The boundary equilibrium $E^{*}$.

(a) Consider the poultry subsystem in farms and define a Lyapunov function

$$
V_{21}=S_{f a}-S_{f a}^{*}-S_{f a}^{*} \ln \left(\frac{S_{f a}}{S_{f a}^{*}}\right)+I_{f a}
$$

and then the derivative of $V_{21}$ is

$$
\frac{d V_{21}}{d t}=\frac{S_{f a}-S_{f a}^{*}}{S_{f a}} \frac{d S_{f a}}{d t}+\frac{d I_{f a}}{d t}
$$

Substituting $A=S_{f a}^{*}\left(d_{a}+a\right)$, (2) and (3) into (37), we have

$$
\frac{d V_{21}}{d t}=-\frac{\left(S_{f a}-S_{f a}^{*}\right)^{2}}{S_{f a}}\left(d_{a}+a\right)+\beta_{a} S_{f a}^{*} I_{f a}-\left(d_{a}+a+\alpha_{a}\right) I_{f a}
$$

Substituting $S_{f a}^{*}$ from (24) into the second term of the right hand side of (38)

$$
\frac{d V_{21}}{d t}=-\frac{\left(d_{a}+a\right)}{S_{f a}}\left(S_{f a}-S_{f a}^{*}\right)^{2}+\left(d_{a}+a+\alpha_{a}\right)\left(R_{01}-1\right) I_{f a}
$$


and if $R_{01}<1, \frac{d V_{21}}{d t} \leq 0$, then

$$
\Omega_{4}=\left\{\left(S_{f a}, I_{f a}\right) \in \mathbb{R}_{+}^{2}: \frac{d V_{21}}{d t}=0\right\}=\left\{\left(S_{f a}, I_{f a}\right) \in \mathbb{R}_{+}^{2}: S_{f a}=S_{f a}^{*} I_{f a}=0\right\}=\left\{E_{f a}^{*}\right\} .
$$

By Lassale's invariance principle, $E_{f a}^{*}$ is globally asymptotically stable.

(b) Next, considering the poultry subsystem of markets with the avian components of farms already at the disease-free steady state.

$$
\begin{aligned}
\frac{d S_{m a}}{d t} & =a S_{f a}^{*}-\beta_{m} S_{m a} I_{m a}-d_{m} S_{m a} \\
\frac{d I_{m a}}{d t} & =\beta_{m} S_{m a} I_{m a}-d_{m} I_{m a}-\alpha_{m} I_{m a} .
\end{aligned}
$$

We define a Lyapunov function

$$
V_{22}=S_{m a}-S_{m a}^{*}-S_{m a}^{*} \ln \left(\frac{S_{m a}}{S_{m a}^{*}}\right)+I_{m a}-I_{m a}^{*}-I_{m a}^{*}\left(\frac{I_{m a}}{I_{m a}^{*}}\right),
$$

and then the derivative of $V_{22}$ is

$$
\frac{d V_{22}}{d t}=\frac{S_{m a}-S_{m a}^{*}}{S_{m a}} \frac{d S_{m a}}{d t}+\frac{I_{m a}-I_{m a}^{*}}{I_{m a}} \frac{d I_{m a}}{d t},
$$

and we can deduce that from (24) and (25) that

$$
a S_{f a}^{*}=\beta_{m} S_{m a}^{*} I_{m a}^{*}+d_{m} S_{m a}^{*} \text { and } d_{m}+\alpha_{m}=\beta_{m} S_{m a}^{*} .
$$

Substituting (39) and (40) into (41), then using (42) and rearranging, we have

$$
\frac{d V_{22}}{d t}=\left(2-\frac{S_{m a}^{*}}{S_{m a}}-\frac{S_{m a}}{S_{m a}^{*}}\right)\left(d_{m} S_{m a}^{*}+d_{m} S_{m a}^{*}\left(R_{02}-1\right)\right)
$$

Since $2-\frac{S_{m a}^{*}}{S_{m a}}-\frac{S_{m a}}{S_{m a}^{*}} \leq 0$, if $R_{01}>1, \frac{d V_{22}}{d t} \leq 0$, thus,

$$
\begin{aligned}
\Omega_{5} & =\left\{\left(S_{m a}, I_{m a}\right) \in R_{+}^{2}: d V_{22} / d t=0\right\} \\
& =\left\{\left(S_{m a}, I_{m a}\right) \in R_{+}^{2}: S_{m a}=S_{m a}^{*}, I_{m a}=I_{m a}^{*}\right\}=\left\{E_{m a}^{*}\right\} .
\end{aligned}
$$

According to Lassalle's invariance principle, $E_{m a}^{*}$ is globally asymptotically stable.

(c) Finally, considering the human subsystem with the avian components of markets already at the endemic steady state

$$
\left.\begin{array}{c}
\frac{d S_{h}}{d t}=A_{h}-\beta_{h} S_{h} I_{m a}^{*}-d_{h} S_{h}+\left(1-d_{h}\right) R_{h}, \\
\frac{d I_{h}}{d t}=\beta_{h} S_{h} I_{m a}^{*}-d_{h} I_{h}-r I_{h}-\alpha_{h} I_{h}, \\
\frac{d R_{h}}{d t}=r I_{h}-R_{h} .
\end{array}\right\}
$$

We define a Lyapunov function

$$
V_{23}=S_{h}-S_{h}^{*}-S_{h}^{*} \ln \frac{S_{h}}{S_{h}^{*}}+I_{h}-I_{h}^{*}-I_{h}^{*} \ln \frac{I_{h}}{I_{h}^{*}}+R_{h}-R_{h}^{*}-R_{h}^{*} \ln \frac{R_{h}}{R_{h}^{*}}
$$

and then the derivative of $V_{23}$ is

$$
\frac{d V_{23}}{d t}=\frac{S_{h}-S_{h}^{*}}{S_{h}} \frac{d S_{h}}{d t}+\frac{I_{h}-I_{h}^{*}}{I_{h}} \frac{d I_{h}}{d t}+\frac{R_{h}-R_{h}^{*}}{R_{h}} \frac{d R_{h}}{d t},
$$

and from (27), we can deduce

$$
\left.\begin{array}{c}
A_{h}=\beta_{h} S_{h}^{*} I_{m a}^{*}+d_{h} S_{h}^{*}-\left(1-d_{h}\right) R_{h}, \\
d_{h}+r+\alpha_{h}=\beta_{h} S_{h}^{*} \frac{I_{m a}^{* a}}{I_{h}^{*}}, \\
r=\frac{R_{h}^{*}}{I_{h}^{*}} .
\end{array}\right\}
$$


Substituting (43) into (44), then using (45) and rearranging, we have that

$$
\begin{aligned}
\frac{d V_{23}}{d t} & =d_{h} S_{h}^{*}\left(2-\frac{S_{h}^{*}}{S_{h}}-\frac{S_{h}}{S_{h}^{*}}+\frac{R_{h}}{S_{h}}-\frac{R_{h}}{S_{h}^{*}}+\frac{R_{h}^{*}}{S_{h}^{*}}-\frac{R_{h}^{*}}{S_{h}}\right) \\
& +\beta_{h} S_{h}^{*} I_{m a}^{*}\left(3-\frac{S_{h}^{*}}{S_{h}}-\frac{I_{h}}{I_{h}^{*}}-\frac{S_{h} I_{h}^{*}}{S_{h}^{*} I_{h}}\right)+R_{h}^{*}\left(\frac{S_{h}^{*}}{S_{h}}-\frac{S_{h}^{*} R_{h}}{S_{h} R_{h}^{*}}-\frac{R_{h}^{*} I_{h}}{R_{h} I_{h}^{*}}+\frac{I_{h}}{I_{h}^{*}}\right) .
\end{aligned}
$$

Substituting $I_{m a}^{*}$ from (26) into (46), we have

$$
\begin{aligned}
\frac{d V_{23}}{d t}= & d_{h} S_{h}^{*}\left(2-\frac{S_{h}^{*}}{S_{h}}-\frac{S_{h}}{S_{h}^{*}}+\frac{R_{h}}{S_{h}}-\frac{R_{h}}{S_{h}^{*}}+\frac{R_{h}^{*}}{S_{h}^{*}}-\frac{R_{h}^{*}}{S_{h}}\right) \\
& +\frac{d_{m}\left(R_{02}-1\right)}{\beta_{m}} \beta_{h} S_{h}^{*}\left(3-\frac{S_{h}^{*}}{S_{h}}-\frac{I_{h}}{I_{h}^{*}}-\frac{S_{h} I_{h}^{*}}{S_{h}^{*} I_{h}}\right) \\
& +R_{h}^{*}\left(\frac{S_{h}^{*}}{S_{h}}-\frac{S_{h}^{*} R_{h}}{S_{h} R_{h}^{*}}-\frac{R_{h}^{*} I_{h}}{R_{h} I_{h}^{*}}+\frac{I_{h}}{I_{h}^{*}}\right) .
\end{aligned}
$$

Since

$$
\begin{aligned}
& 2-\frac{S_{h}^{*}}{S_{h}}-\frac{S_{h}}{S_{h}^{*}}+\frac{R_{h}}{S_{h}}-\frac{R_{h}}{S_{h}^{*}}+\frac{R_{h}^{*}}{S_{h}^{*}}-\frac{R_{h}^{*}}{S_{h}} \leq 0, \\
& 3-\frac{S_{h}^{*}}{S_{h}}-\frac{I_{h}}{I_{h}^{*}}-\frac{S_{h} I_{h}^{*}}{S_{h}^{*} I_{h}} \leq 0, \\
& \frac{S_{h}^{*}}{S_{h}}-\frac{S_{h}^{*} R_{h}}{S_{h} R_{h}^{*}}-\frac{R_{h}^{*} I_{h}}{R_{h} I_{h}^{*}}+\frac{I_{h}}{I_{h}^{*}} \leq 0
\end{aligned}
$$

if $R_{01}>1, \frac{d V_{23}}{d t} \leq 0$, thus,

$$
\begin{aligned}
\Omega_{6} & =\left\{\left(S_{h}, I_{h}, R_{h}\right) \in \mathbb{R}_{+}^{3}: \frac{d V_{23}}{d t}=0\right\} \\
& =\left\{\left(S_{h}, I_{h}, R_{h}\right) \in \mathbb{R}_{+}^{3}: S_{h}=S_{h}^{*}, I_{h}=I_{h}^{*}, R_{h}=R_{h}^{*}\right\}=\left\{E_{h}^{*}\right\} .
\end{aligned}
$$

According to Lassalle's invariance principle, $E_{h}^{*}$ is globally asymptotically stable. In conclusion, if $R_{01}<1, R_{02}>1$, the boundary equilibrium $E^{*}$ is globally asymptotically stable.

2. The endemic equilibrium $E^{* *}$.

(a) Consider the poultry subsystem in farms and define a Lyapunov function

$$
V_{31}=S_{f a}-S_{f a}^{* *}-S_{f a}^{* *} \ln \frac{S_{f a}}{S_{f a}^{* *}}+I_{f a}-I_{f a}^{* *}-I_{f a}^{* *} \ln \frac{I_{f a}}{I_{f a}^{* *}},
$$

and then the derivative of $V_{31}$ along solutions of system (5) is

$$
\frac{d V_{31}}{d t}=\frac{S_{f a}-S_{f a}^{* *}}{S_{f a}} \frac{d S_{f a}}{d t}+\frac{I_{f a}-I_{f a}^{* *}}{I_{f a}} \frac{d I_{f a}}{d t} .
$$

We can deduce that $a+\alpha_{a}+d_{a}=\beta_{a} S_{f a}^{* *}$ and $A_{a}=\beta_{a} S_{f a}^{* *} I_{f a}^{* *}+\left(a+d_{a}\right) S_{f a}^{* *}$ from (17) and (18) and on substituting into (47), we obtain

$$
\frac{d V_{31}}{d t}=-\frac{\left(a+d_{a}\right)}{S_{f a}}\left(S_{f a}-S_{f a}^{* *}\right)^{2}+\beta_{a} S_{f a}^{* *} I_{f a}^{* *}\left(2-\frac{S_{f a}^{* *}}{S_{f a}}-\frac{S_{f a}}{S_{f a}^{* *}}\right) .
$$

Substituting $I_{f a}^{* *}$ from (18) into (48) we have,

$$
\frac{d V_{31}}{d t}=-\frac{\left(a+d_{a}\right)}{S_{f a}}\left(S_{f a}-S_{f a}^{* *}\right)^{2}+S_{f a}^{* *}\left(a+d_{a}\right)\left(R_{01}-1\right)\left(2-\frac{S_{f a}^{* *}}{S_{f a}}-\frac{S_{f a}}{S_{f a}^{* *}}\right) .
$$


If $R_{01}>1$, then $2-\frac{S_{f a}^{* *}}{S_{f a}}-\frac{S_{f a}}{S_{f a}^{* *}} \leq 0$ and $\frac{d V_{31}}{d t} \leq 0$ and thus,

$$
\begin{aligned}
\Omega_{7} & =\left\{\left(S_{f a}, I_{f a}\right) \in R_{+}^{2}: \frac{d V_{31}}{d t}=0\right\} \\
& =\left\{\left(S_{f a}, I_{f a}\right) \in R_{+}^{2}: S_{f a}=S_{f a}^{* *}, I_{f a}=I_{f a}^{* *}\right\}=\left\{E_{f a}^{* *}\right\} .
\end{aligned}
$$

According to Lassalle's invariance principle, $E_{h}^{*}$ is globally asymptotically stable.

(b) Consider the poultry subsystem of markets with the avian components of farms already at the endemic steady state;

$$
\begin{aligned}
\frac{d S_{m a}}{d t} & =a S_{f a}^{* *}-\beta_{m} S_{m a} I_{m a}-d_{m} S_{m a} \\
\frac{d I_{m a}}{d t} & =a I_{f a}^{* *}+\beta_{m} S_{m a} I_{m a}-d_{m} I_{m a}-\alpha_{m} I_{m a}
\end{aligned}
$$

we define a Lyapunov function

$$
V_{32}=S_{m a}-S_{m a}^{* *}-S_{m a}^{* *} \ln \frac{S_{m a}}{S_{m a}^{* *}}+I_{m a}-I_{m a}^{* *}-I_{m a}^{* *} \ln \frac{I_{m a}}{I_{m a}^{* *}}
$$

and then the derivative of $V_{32}$ is

$$
\frac{d V_{32}}{d t}=\frac{S_{m a}-S_{m a}^{* *}}{S_{m a}} \frac{d S_{m a}}{d t}+\frac{I_{m a}-I_{m a}^{* *}}{I_{m a}} \frac{d I_{m a}}{d t} .
$$

Substitute Equations (49) and (50). Putting the relation $a S_{f a}^{* *}=d_{m} S_{m a}^{* *}+\beta_{m} S_{m a}^{* *} I_{m a}^{* *}$ and $d_{m}+\alpha_{m}=$ $\beta_{m} S_{m a}^{* *}+\frac{a I_{f a}^{* *}}{I_{m a}^{* *}}\left(\right.$ which can be easily obtained from (17) and (22)) and substituting $I_{f a}^{* *}$ from (18) and then rearranging, we have

$$
\begin{aligned}
\frac{d V_{32}}{d t} & =\frac{a\left(a+d_{a}\right)\left(R_{01}-1\right)}{\beta_{a}}\left(2-\frac{I_{m a}^{* *}}{I_{m a}}-\frac{I_{m a}}{I_{m a}^{* *}}\right) \\
& +d_{m} S_{m a}^{* *}\left(2-\frac{S_{m a}^{* *}}{S_{m a}}-\frac{S_{m a}}{S_{m a}^{* *}}\right)+\beta_{m} S_{m a}^{* *} I_{m a}^{* *}\left(2-\frac{S_{m a}^{* *}}{S_{m a}}-\frac{S_{m a}}{S_{m a}^{* *}}\right)
\end{aligned}
$$

Clearly, if $R_{01}>1, R_{02}>1$, then

$$
2-\frac{S_{m a}^{* *}}{S_{m a}}-\frac{S_{m a}}{S_{m a}^{* *}} \leq 0,2-\frac{I_{m a}^{* *}}{I_{m a}}-\frac{I_{m a}}{I_{m a}^{* *}} \leq 0
$$

and $\frac{d V_{32}}{d t} \leq 0$. Thus,

$$
\begin{aligned}
\Omega_{8} & =\left\{\left(S_{m a}, I_{m a}\right) \in \mathbb{R}_{+}^{2}: \frac{d V_{32}}{d t}=0\right\} \\
& =\left\{\left(S_{m a}, I_{m a}\right) \in \mathbb{R}_{+}^{2}: S_{m a}=S_{m a}^{* *}, I_{m a}=I_{m a}^{* *}\right\}=\left\{E_{m a}^{* *}\right\} .
\end{aligned}
$$

According to Lassalle's invariance principle, $E_{m a}^{* *}$ is globally asymptotically stable.

(c) Finally, we consider the human subsystem with the avian components already at the endemic steady state

$$
\left.\begin{array}{c}
\frac{d S_{h}}{d t}=A_{h}-\beta_{h} S_{h} I_{m a}^{* *}-d_{h} S_{h} \\
\frac{d I_{h}}{d t}=\beta_{h} S_{h} I_{m a}^{* *}-d_{h} I_{h}-r I_{h}-\alpha_{h} I_{h} \\
\frac{d R_{h}}{d t}=r I_{h}-R_{h}
\end{array}\right\}
$$

We define a Lyapunov function

$$
V_{33}=S_{h}-S_{h}^{* *}-S_{h}^{* *} \ln \frac{S_{h}}{S_{h}^{* *}}+I_{h}-I_{h}^{* *}-I_{h}^{* *} \ln \frac{I_{h}}{I_{h}^{* *}}+R_{h}-R_{h}^{* *}-R_{h}^{* *} \ln \frac{R_{h}}{R_{h}^{* *}}
$$

and then the derivative of $V_{33}$ is given as

$$
\frac{d V_{33}}{d t}=\frac{S_{h}-S_{h}^{* *}}{S_{h}} \frac{d S_{h}}{d t}+\frac{I_{h}-I_{h}^{* *}}{I_{h}} \frac{d I_{h}}{d t}+\frac{R_{h}-R_{h}^{* *}}{R_{h}} \frac{d R_{h}}{d t}
$$


Using the relations $A_{h}=d_{h} S_{h}^{* *}+\beta_{h} S_{h}^{* *} I_{m a}^{* *}-\left(1-d_{h}\right) R_{h}^{* *}, d_{h}+r+\alpha_{h}=\beta_{h} S_{h}^{* *} \frac{I_{m a}^{* * a}}{I_{h}^{* *}}$ and $r=R_{h}^{* *} / I_{h}^{* *}$ (which can be easily obtained from (23)), we have

$$
\begin{aligned}
\frac{d V_{33}}{d t}= & d_{h} S_{h}^{* *}\left(2-\frac{S_{h}^{* *}}{S_{h}}-\frac{S_{h}}{S_{h}^{* *}}+\frac{R_{h}}{S_{h}}-\frac{R_{h}}{S_{h}^{* *}}+\frac{R_{h}^{* *}}{S_{h}^{* *}}-\frac{R_{h}^{* *}}{S_{h}}\right) \\
& +\beta_{h} S_{h}^{* *} I_{m a}^{* *}\left(3-\frac{S_{h}^{* *}}{S_{h}}-\frac{I_{h}}{I_{h}^{* *}}-\frac{S_{h} I_{h}^{* *}}{S_{h}^{* *} I_{h}}\right) \\
& +R_{h}^{* *}\left(\frac{S_{h}^{* *}}{S_{h}}-\frac{S_{h}^{* *} R_{h}}{S_{h} R_{h}^{* *}}-\frac{R_{h}^{* *} I_{h}}{R_{h} I_{h}^{* *}}+\frac{I_{h}}{I_{h}^{* *}}\right)
\end{aligned}
$$

Since if $R_{01}>1$, then

$$
\begin{aligned}
& 2-\frac{S_{h}^{* *}}{S_{h}}-\frac{S_{h}}{S_{h}^{* *}}+\frac{R_{h}}{S_{h}}-\frac{R_{h}}{S_{h}^{* *}}+\frac{R_{h}^{* *}}{S_{h}^{* *}}-\frac{R_{h}^{* *}}{S_{h}} \leq 0 \\
& 3-\frac{S_{h}^{* *}}{S_{h}}-\frac{I_{h}}{I_{h}^{* *}}-\frac{S_{h} I_{h}^{* *}}{S_{h}^{* *} I_{h}} \leq 0 \\
& \frac{S_{h}^{* *}}{S_{h}}-\frac{S_{h}^{* *} R_{h}}{S_{h} R_{h}^{* *}}-\frac{R_{h}^{* *} I_{h}}{R_{h} I_{h}^{* *}}+\frac{I_{h}}{I_{h}^{* *}} \leq 0
\end{aligned}
$$

and $\frac{d V_{33}}{d t} \leq 0$. Thus,

$$
\begin{aligned}
\Omega_{9} & =\left\{\left(S_{h}, I_{h}, R_{h}\right) \in R_{+}^{3}: d V_{33} / d t=0\right\} \\
& =\left\{\left(S_{h}, I_{h}, R_{h}\right) \in R_{+}^{3}: S_{h}=S_{h}^{* *}, I_{h}=I_{h}^{* *}, R_{h}=R_{h}^{* *}\right\}=\left\{E_{h}^{* *}\right\} .
\end{aligned}
$$

According to Lassalle's invariance principle, $E_{h}^{* *}$ is globally asymptotically stable.

Remark 1. Stability of the equilibrium depends on the Lyapunov functions. The quadratic form of Lyapunov functions is usually used in most references [21,22]. Let us take the function in this paper

$$
f(x)=1-x+\ln x, \forall x>0,
$$

we have

$$
f(1)=0, f^{\prime}(x)=\frac{1}{x}-1,
$$

thus, $f(x)=1-x+\ln x \leq 0 \forall x>0$, and the equality holds only when $x=1$. Let $x=\frac{S_{f a}}{S_{f a}^{0}}>0$, then $S_{f a}-$ $S_{f a}^{0}-S_{f a}^{0} \ln \left(\frac{S_{f a}}{S_{f a}^{0}}\right) \geq 0$. Hence, $V_{11}$ is a Lyapunov function. In a similar way, $V_{12}, V_{13}, V_{21}, V_{22}, V_{23}, V_{31}, V_{32}, V_{33}$ are also Lyapunov functions.

\section{Numerical Simulation}

The existence of the disease free equilibrium, the boundary equilibrium, and the endemic equilibrium of system (2-8) have been established in the previous sections. In what follows, we simulate the stability of these equilibria by plotting the graphs of the system (2-8) for different values of the parameters corresponding to each of these equilibria. Figure 1 verifies that the disease dies out of the population and Figures 2 and 3 indicate that the disease is sustained in the population.

Tables (1-4) show the effect of disease-related death rates of infected poultry of markets $\alpha_{m}$, the disease-related death rates of infected poultry of farms $\alpha_{a}$, the transmission coefficient from infective poultry of farms to susceptible poultry of farms $\beta_{a}$ and the contact rate from infective poultry of markets to susceptible poultry of markets $\beta_{m}$ on the reproduction number. As can be seen in Tables 1 and 2, increase in $\alpha_{m}$ and $\alpha_{a}$ lead to decrease in $R_{02}$ and $R_{01}$ respectively. Increase in $\beta_{a}$ and $\beta_{m}$ lead to an increase in $R_{01}$ and $R_{02}$ respectively (see Tables 3 and 4 . 

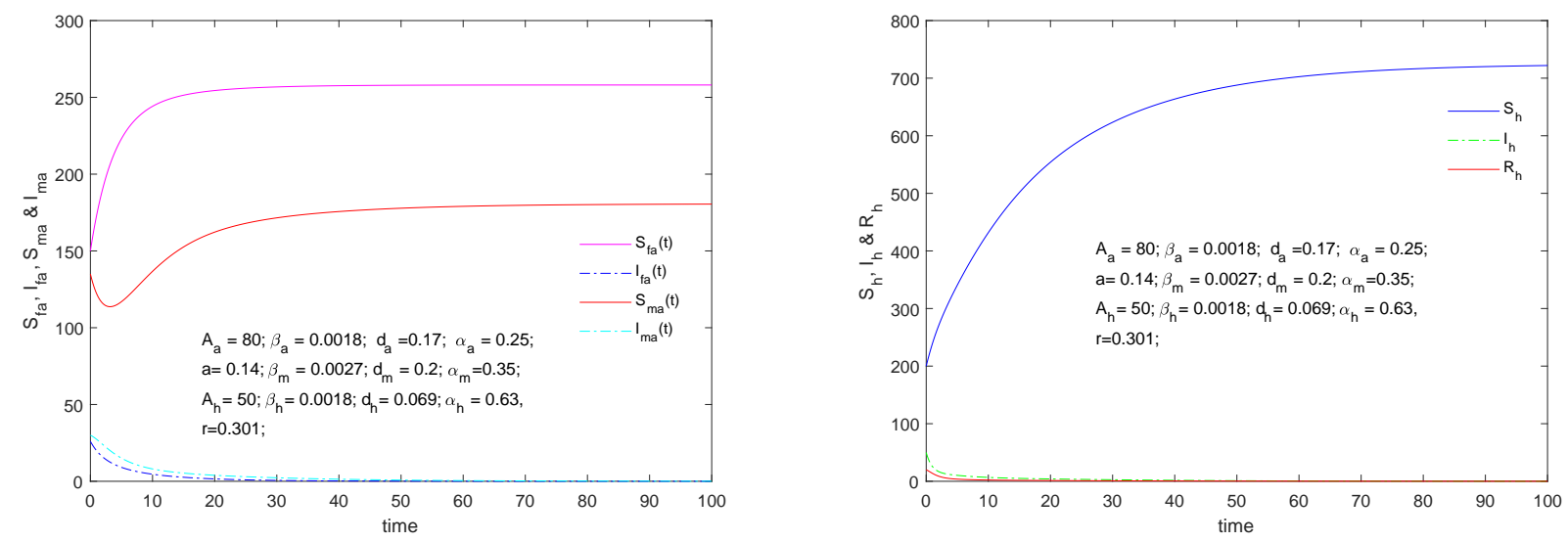

Figure 1. Time-variation diagram of system (2-8) when $R_{0}=0.88680353<1$.
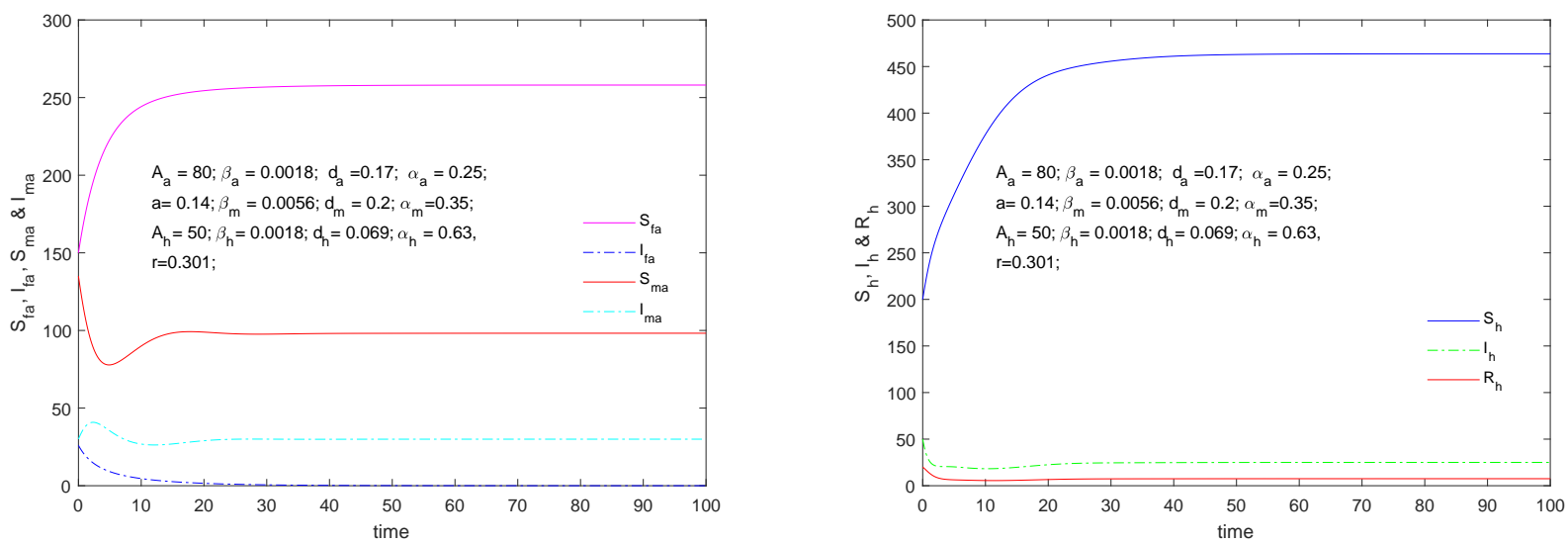

Figure 2. Time-variation diagram of system (2-8) when $R_{01}=0.82949309<1$ and $R_{02}=1.83929618>1$.
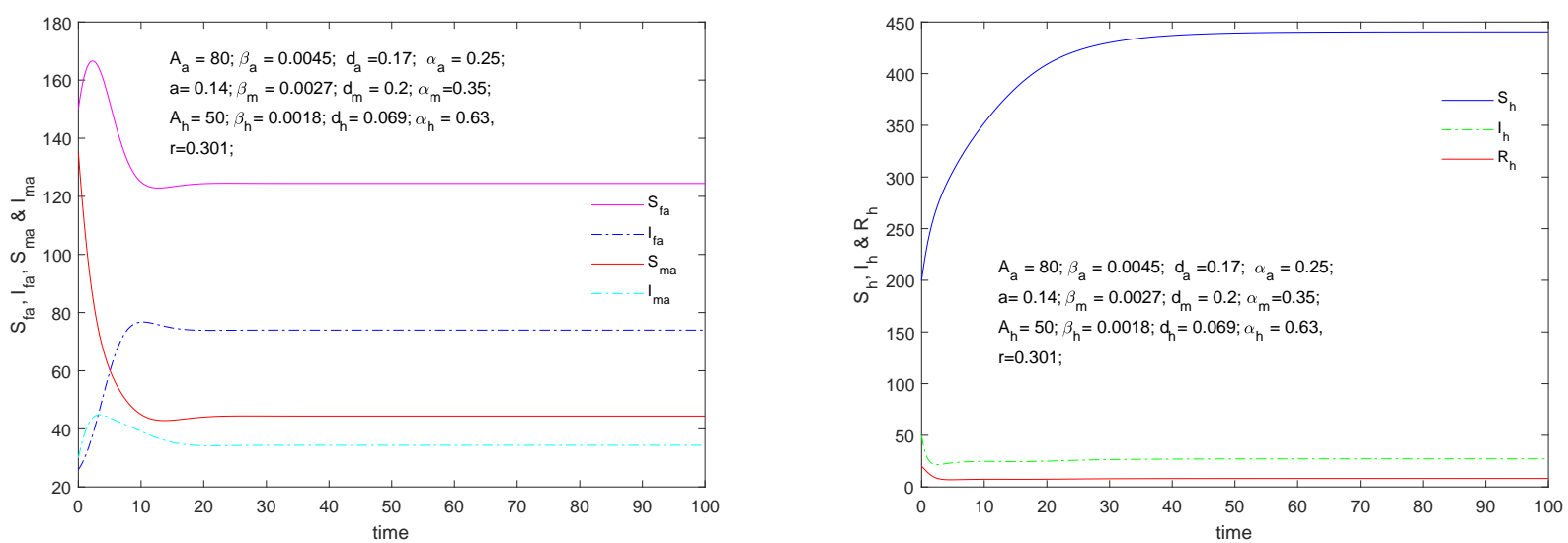

Figure 3. Time-variation diagram of system (2-8) when $R_{01}=2.07373271>1$ and $R_{02}=1.83929618>1$. 
Table 1. Table showing the effect of $\alpha_{m}$ on the reproduction number

\begin{tabular}{|c|c|c|c|c|c|c|c|}
\hline$\alpha_{m}$ & 0.0500 & 0.2000 & 0.3500 & 0.5000 & 0.650 & 0.8000 & 0.9500 \\
\hline$R_{01}$ & 2.0737 & 2.0737 & 2.0737 & 2.0737 & 2.0737 & 2.0737 & 2.0737 \\
\hline$R_{02}$ & 4.0465 & 2.5290 & 1.8393 & 1.4452 & 1.1901 & 1.0116 & 0.8797 \\
\hline
\end{tabular}

Table 2. Table showing the effect of $\alpha_{a}$ on the reproduction number

\begin{tabular}{|c|c|c|c|c|c|c|c|}
\hline$\alpha_{a}$ & 0.0500 & 0.2000 & 0.3500 & 0.5000 & 0.650 & 0.8000 & 0.9500 \\
\hline$R_{01}$ & 3.2258 & 2.2770 & 1.7595 & 1.4337 & 1.2097 & 1.0462 & 0.9217 \\
\hline$R_{02}$ & 1.8393 & 1.8393 & 1.8393 & 1.8393 & 1.8393 & 1.8393 & 1.8393 \\
\hline
\end{tabular}

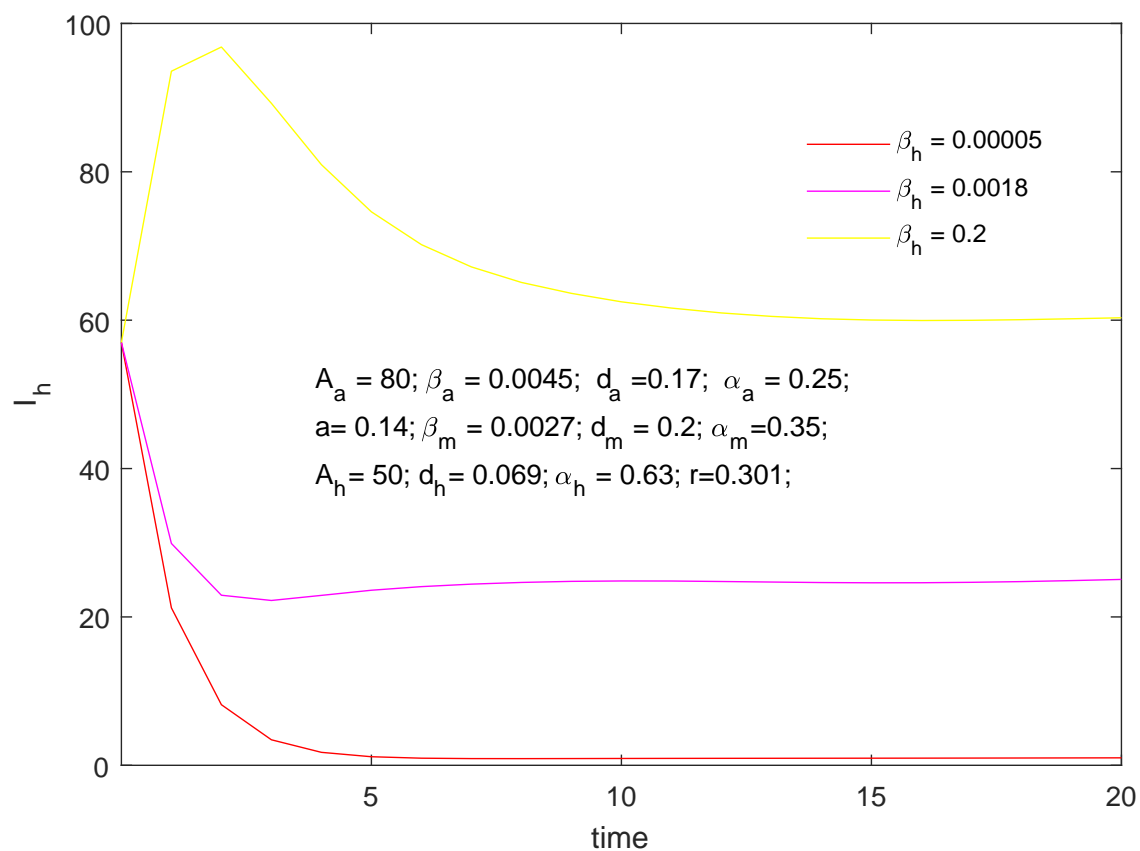

Figure 4. The curve-trend diagram of $I_{h}$ with time, as $\beta_{h}$ varies.

\section{Discussion}

We modify the SI-SI-SIR model developed for Avian influenza infectious diseases by [1] by including human re-infection. We obtain the reproduction number $R_{0}=\max \left\{R_{01}, R_{02}\right\}$ which is enough to predict whether the the spread becomes endemic or not as follows;

1. If $R_{0}<1$ (i.e. $R_{01}<1$ and $R_{02}<1$ ), there exists only the disease free equilibrium. We showed the disease free equilibrium is globally asymptotically stable. Hence, the disease dies out (see Figure 1).

2. If $R_{01}<1$ and $R_{02}>1$, (i.e. $R_{0}>1$ ), there exists only the boundary equilibrium. We showed that the boundary equilibrium is globally asymptotically stable. The disease will be sustained in the population and eventually lead to epidemic (see Figure 2).

3. If $R_{01}>1$ and $R_{02}>1$, (i.e. $R_{0}>1$ ), there exists only the endemic equilibrium. We showed that the endemic equilibrium is globally asymptotically stable and this means the disease will spread (see Figure $3)$.

Table 3. Table showing the effect of $\beta_{a}$ on the reproduction number

\begin{tabular}{|c|c|c|c|c|c|c|c|}
\hline$\beta_{a}$ & 0.0045 & 0.1545 & 0.3045 & 0.4545 & 0.6045 & 0.7545 & 0.9045 \\
\hline$R_{01}$ & 2.0737 & 71.1982 & 140.3226 & 209.4470 & 278.5714 & 347.6959 & 416.8203 \\
\hline$R_{02}$ & 1.8393 & 1.8393 & 1.8393 & 1.8393 & 1.8393 & 1.8393 & 1.8393 \\
\hline
\end{tabular}


Table 4. Table showing the effect of $\beta_{m}$ on the reproduction number

\begin{tabular}{|c|c|c|c|c|c|c|c|}
\hline$\beta_{m}$ & 0.0056 & 0.1556 & 0.3056 & 0.4556 & 0.6056 & 0.7556 & 0.9056 \\
\hline$R_{01}$ & 2.0737 & 2.0737 & 2.0737 & 2.0737 & 2.0737 & 2.0737 & 2.0737 \\
\hline$R_{02}$ & 1.8393 & 51.1062 & 100.3730 & 149.6399 & 198.9067 & 248.1736 & 297.4405 \\
\hline
\end{tabular}

4. If $R_{01}>1$ and $R_{02}<1$, (i.e. $R_{0}>1$ ), there exists no positive equilibrium.

In the case of reinfection, as included in this paper, it is observed that the number of susceptible human increase more than the case of no re-infection (as the case in [1]). This can be traced to the fact that a fraction of the recovered human (i.e. those who do not die naturally) move back into the susceptible class. In order to reduce the spread of the avian influenza, it is observed from Table 1, Table 2, Table 3, Table 4 and Figure 4 that the following measures can be taken;

1. increase $\alpha_{a}$ and $\alpha_{m}$. This can be achieved by killing of infected poultry.

2. reduce $\beta_{a}, \beta_{m}$ and $\beta_{h}$. This can be achieved by closing down some farms and markets where infection is detected.

It is important to note that our results agree with the results obtained in [1].

The two authors jointly developed the model. The first author did the dynamical analysis and the second author did the simulation and arranged the document.

Acknowledgments: We acknowledge Adekunle Ajasin University, Akungba Akoko for the unlimited access to their facilities.

Author Contributions: All authors contributed equally to the writing of this paper. All authors read and approved the final manuscript.

Conflicts of Interest: "The authors declare no conflict of interest."

\section{References}

[1] Li, Y., Qin, P., \& Zhang, J. (2018). Dynamics analysis of avian influenza A (H7N9) epidemic model. Discrete Dynamics in Nature and Society, 2018, Article ID 7485310, 12 pages.

[2] Spackman, E., Pantin-Jackwood, M., Swayne, D. E., Suarez, D. L., \& Kapczynski, D. R. (2015). Impact of route of exposure and challenge dose on the pathogenesis of H7N9 low pathogenicity avian influenza virus in chickens. Virology, 477, 72-81.

[3] Wang, C., Wang, J., Su, W., Gao, S., Luo, J., Zhang, M., ... \& Jia, Y. (2013). Relationship between domestic and wild birds in live poultry market and a novel human H7N9 virus in China. The Journal of infectious diseases, 209(1), 34-37.

[4] Kageyama, T., Fujisake, S., Takashita, E., Xu, H., \& Yamada, S. (2013). Genetic analysis of novel avian A (H7N9) influenza viruses isolated from patients in China, February to April 2013. Eurosurveillance 18, 1Ú6.

[5] Xiong, C., Zhang, Z., Jiang, Q., \& Chen, Y. (2013). Evolutionary characteristics of A/Hangzhou/1/2013 and source of avian influenza virus H7N9 subtype in China. Clinical infectious diseases, 57(4), 622-624.

[6] Zhou, J., Wang, D., Gao, R., Zhao, B., Song, J., Qi, X., ... \& Bai, T. (2013). Biological features of novel avian influenza A (H7N9) virus. Nature, 499(7459), 500.

[7] Teng, Y., Bi, D., Guo, X., Hu, D., Feng, D., \& Tong, Y. (2018). Contact reductions from live poultry market closures limit the epidemic of human infections with H7N9 influenza. Journal of Infection, 76(3), 295-304.

[8] Nokkaew, A., Modchang, C., Amornsamankul, S., Lenbury, Y., Pimpunchat, B., \& Triampo, W. (2017). Mathematical modeling of infectious disease transmission in macroalgae. Advances in Difference Equations, 2017(1), 288.

[9] Iwami, S., Takeuchi, Y., \& Liu, X. (2007). AvianŰhuman influenza epidemic model. Mathematical biosciences, 207(1), $1-25$.

[10] Che, S., Xue, Y., \& Ma, L. (2014). The stability of highly pathogenic avian influenza epidemic model with saturated contact rate. Applied Mathematics, 5(21), 3365.

[11] Liu, Z., \& Fang, C. T. (2015). A modeling study of human infections with avian influenza A H7N9 virus in mainland China. International Journal of Infectious Diseases, 41, 73-78.

[12] Khan, M. A., Farhan, M., Islam, S., \& Bonyah, E. (2018). Modeling the transmission dynamics of avian influenza with saturation and psychological effect. Discrete \& Continuous Dynamical Systems-S, 12(3), 455.

[13] Zhang, J., Li, Y., Jin, Z., \& Zhu, H. (2019). Dynamics Analysis of an Avian Influenza A (H7N9) Epidemic Model with Vaccination and Seasonality. Complexity, 2019.

[14] Bidari, S., \& Goldwyn, E. E. (2019). Stochastic models of influenza outbreaks on a college campus. Letters in Biomathematics, 1-14. 
[15] Kharis, M., \& Arifudin, R. (2017). Mathematical Model of Seasonal Influenza with Treatment in Constant Population. In Journal of Physics: Conference Series (Vol. 824(1), 012034). IOP Publishing.

[16] Hill, E. M., Petrou, S., De Lusignan, S., Yonova, I., \& Keeling, M. J. (2019). Seasonal influenza: Modelling approaches to capture immunity propagation. BioRxiv, 637074.

[17] Van den Driessche, P., \& Watmough, J. (2002). Reproduction numbers and sub-threshold endemic equilibria for compartmental models of disease transmission. Mathematical biosciences, 180(1-2), 29-48.

[18] Oke, A. S. Validation of Kaplan-Yorke Conjecture for some Attractors. Journal of Nigerian Association of Mathematical Physics, 28(1),47-50.

[19] LaSalle, J. P. (1968). Stability theory for ordinary differential equations. Journal of Differential Equations, 4(1), 57-65.

[20] LaSalle, J. P. (1976). The stability of dynamical systems (Vol. 25). Siam, America.

[21] Oke, A. S., Akintewe, S. M., \& Akinbande, A. G. (2018). Generalized Euclidean Least Square Approximation. Asian Journal of Probability and Statistics, 1-10.

[22] Oke, A. S. (2017). Convergence of Differential Transform Method for Ordinary Differential Equations. Journal of Advances in Mathematics and Computer Science, 24(6), 1-17.

(C) 2019 by the authors; licensee PSRP, Lahore, Pakistan. This article is an open access article distributed under the terms and conditions of the Creative Commons Attribution (CC-BY) license (http://creativecommons.org/licenses/by/4.0/). 